EPJ Web of Conferences 23, 00012 (2012)

DOI: $10.1051 /$ epjconf/20122300012

(C) Owned by the authors, published by EDP Sciences, 2012

\title{
Superconductivity, spin and charge order, and quantum criticality in correlated electron materials
}

\author{
M. B. Maple ${ }^{1}$, J. J. Hamlin ${ }^{1}$, D. A. Zocco ${ }^{1}$, M. Janoschek ${ }^{1,}$, R. E. Baumbach ${ }^{1,{ }^{*}}$, B. D. White ${ }^{1}$, I. R. Fisher ${ }^{2}$, and J.-H. Chu ${ }^{2}$ \\ ${ }^{1}$ Department of Physics, University of California, San Diego, La Jolla, California 92093 USA \\ ${ }^{2}$ Department of Applied Physics, Geballe Laboratory for Advanced Materials, Stanford University, \\ Stanford, California 94305, USA \\ *Present address: Los Alamos National Laboratory, Los Alamos, New Mexico 87545, USA
}

\begin{abstract}
We describe recent experiments performed in our laboratory that address spin or charge ordered phases in novel rare earth and actinide based materials and phenomena that emerge when these ordered phases are suppressed toward $0 \mathrm{~K}$ by varying an external control parameter such as chemical composition, pressure, or magnetic field. Specific examples discussed include magnetic order, heavy fermion behavior, and unconventional quantum criticality in noncentrosymmetric $M_{2} T_{12} \mathrm{P}_{7}$ compounds ( $M=$ rare earth, actinide; $T=$ $\mathrm{Co}, \mathrm{Fe})$ and the interplay of superconductivity and charge density waves in rare earth tritelluride compounds $R \mathrm{Te}_{3}(R=\mathrm{Gd}, \mathrm{Tb}, \mathrm{Dy})$.
\end{abstract}

\section{Introduction}

Multinary compounds based on transition metal, rare earth, and actinide elements in which the localized $d$ - and $f$-electron states are admixed with conduction electron states provide a wealth of strongly correlated electron phenomena. These phenomena include, for example, metal-insulator transitions, colossal magnetoresistance, multifunctional phenomena, valence fluctuations, hybridization gap semiconductivity, heavy fermion behavior, non-Fermi liquid behavior, unconventional superconductivity, magnetic order, and quadrupolar order. The discovery of these correlated electron phenomena is largely the result of the development of new materials and is an example of "materials driven physics" [1].

The occurrence of such a wide range of phenomena arises from a delicate interplay of competing interactions that can often be "tuned" by variation of an external control parameter $\delta$ such as chemical composition $x$, pressure $P$, or magnetic field $H$, resulting in complex temperature $T$ vs $x, P$, and $H$ phase diagrams. A particularly striking phenomenon that has been observed in many correlated electron systems, including heavy fermion $f$-electron compounds, high $T_{\mathrm{c}}$ superconducting cuprates, and, more recently, many Fe-based materials, is the emergence of superconductivity near the critical value $\delta_{\text {cr }}$ of the control parameter (usually, $x_{\text {cr }}$ or $P_{\text {cr }}$ ) where a magnetically ordered phase is suppressed to $0 \mathrm{~K}$. For many systems, the Fermi liquid (FL) paradigm is found to be violated in the vicinity of $\delta_{\text {cr }}$, which is manifested as weak power law and logarithmic divergences in the physical properties at low temperature (so-called nonFermi liquid behavior) [2-9]. The most frequently observed non-Fermi liquid (NFL) characteristics for the electrical resistivity $\rho$, specific heat $C$, magnetic susceptibility $\chi$, and imaginary part of the dynamical susceptibility $\chi$ " are represented by the following expressions:

$\rho(T) \sim \pm A T^{n}(0.5 \leq n \leq 1.5$, but usually $n \sim 1)$

$C(T) / \mathrm{T} \sim-\ln (T)$

$\chi(T)$ - exhibits non-Curie Weiss behavior

$\chi{ }^{\prime}(\omega, T)-$ scales as $\omega / T(\omega$ is frequency $)$

These characteristics seem almost universal and are observed in both stoichiometric and chemically substituted compounds. The superconductivity and the NFL behavior may be due to quantum fluctuations of the magnetic order parameter (OP) associated with the suppression of a second order magnetic phase transition to $0 \mathrm{~K}$ at $\delta_{\text {cr }}$, where $\delta_{\text {cr }}$ is referred to as a quantum critical point (QCP). The formation of the superconducting phase appears to "protect" the QCP by removing the degeneracy associated with the OP fluctuations, and the superconducting electron pairing is apparently mediated

This is an Open Access article distributed under the terms of the Creative Commons Attribution-Noncommercial License 3.0, which permits unrestricted use, distribution, and reproduction in any noncommercial medium, provided the original work is properly cited. 
by magnetic interactions. In contrast, magnetic interactions generally have a destructive effect on conventional BCS superconductivity. Interestingly, superconductivity has been found to emerge from charge ordered phases such as charge density waves (CDW). In this paper, we describe experiments, carried out in our laboratory, that address the interrelation of superconductivity, spin and charge order, and NFL behavior in novel $d$ - and $f$-electron materials. Two examples are considered in this paper: magnetic order, heavy fermion behavior and unconventional quantum criticality in noncentrosymmetric $M_{2} T_{12} \mathrm{P}_{7}(M=$ rare earth, actinide; $T=\mathrm{Co}, \mathrm{Fe}$ ) compounds and the emergence of superconductivity upon the suppression of CDW ordering in the rare earth tritellurides $R \mathrm{Te}_{3}$.

\section{Magnetic order, heavy fermion behavior, and unconventional quantum criticality in noncentrosymmetric $M_{2} T_{12} P_{7}$ ( $M=$ rare earth, actinide; $T=$ Co, Fe) compounds}

Noncentrosymmetric compounds have attracted a great deal of interest in recent years because they may exhibit unconventional superconductivity with mixed singlet and triplet spin pairing [10], exotic types of magnetic order [11] mediated by the Dzyaloshinskii-Moriya interaction [12-13], and unconventional quantum critical behavior [14]. During the past several years, we have been involved in an investigation of the physical properties of $M_{2} T_{12} P n_{7}$ compounds as a function of temperature (down to $40 \mathrm{mK}$ ) and magnetic field (up to $9 \mathrm{~T}$ ) which has yielded some striking examples of correlated electron behavior for the compounds with $M=\mathrm{Sm}, \mathrm{Yb}$, and $\mathrm{U}$. The experiments were performed on single crystals of $M_{2} \mathrm{Fe}_{12} \mathrm{P}_{7}$ compounds that were harvested as byproducts in our efforts to synthesize $M \mathrm{FePO}$ and $M \mathrm{Fe}_{2} \mathrm{P}_{2}(M=$ rare earth, actinide) compounds by growth in a molten Sn flux [15]. The $M_{2} \mathrm{Fe}_{12} \mathrm{P}_{7}$ compounds belong to a large class of compounds with the chemical formula $M_{2} T_{12} P n_{7}$, which have the hexagonal, noncentrosymmetric, $\mathrm{Zr}_{2} \mathrm{Fe}_{12} \mathrm{P}_{7}$-type crystal structure (space group $P$-6) [16] and can be formed with many metal $M$, transition metal $T$, and pnictogen $P n$ elements $(M=\mathrm{Li}, \mathrm{Na}, \mathrm{Ca}, \mathrm{Mg}, \mathrm{Ti}-\mathrm{Hf}, \mathrm{Nb}$, Sc, Y, La-Lu; $T=\mathrm{Mn}, \mathrm{Fe}, \mathrm{Co}, \mathrm{Ni}, \mathrm{Ru} ; P n=\mathrm{P}, \mathrm{As})[17-$ 19]. The $M_{2} T_{12} P n_{7}$ compounds comprise a subclass of a larger class of pnictogen-based compounds with noncentrosymmetric structures and the chemical formula $M_{n(n-1)} T_{(n+1)(n+2)} P n_{n(n+1)+1}[17-18,20]$.

\subsection{Unconventional quantum criticality in $\mathrm{Yb}_{2} \mathrm{Fe}_{12} \mathrm{P}_{7}$}

Our studies of $\mathrm{Yb}_{2} \mathrm{Fe}_{12} \mathrm{P}_{7}$ single crystals by means of electrical resistivity $\rho$, specific heat $C$, and magnetization $M$ measurements revealed an unexpectedly rich variety of low- $T$ phenomena [21]. The $T-H$ phase diagram, shown in Fig. 1, suggests that there is a crossover from a magnetically ordered NFL phase at low $H$ to another
NFL phase at higher $H$, indicating the occurrence of a quantum phase transition between the two NFL phases. The crossover occurs near the value of $H$ where the magnetic ordering temperature $\left(T_{\mathrm{M}}\right)$ is no longer observable in $C(T, H) / T$ and $\rho(T, H)$, but not where $T_{\mathrm{M}}$ extrapolates smoothly to $T=0 \mathrm{~K}$ at a possible QCP. This behavior deviates strongly from the widely accepted QCP scenario where a $2^{\text {nd }}$ order phase transition is suppressed to $T=0 \mathrm{~K}$ at a QCP by varying a control parameter $\delta$. In this picture, order parameter (OP) fluctuations are manifested as NFL behavior at temperatures above the QCP, and a line emanating from the QCP delineates a gradual crossover from NFL to FL behavior at lower $T$ and higher values of $\delta$.

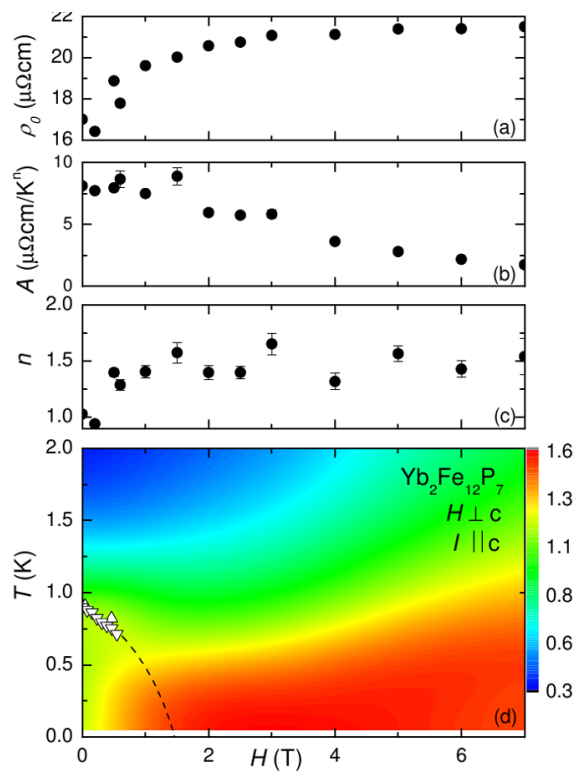

Fig. 1. (a) - (c) Magnetic field $H$ dependence of the quantities $\rho_{0}, A$, and $n$ from power law fits to the electrical resistivity data $\rho(T)$. (d) $T-H$ phase diagram for $\mathrm{Yb}_{2} \mathrm{Fe}_{12} \mathrm{P}_{7}$. The values for $T_{\mathrm{M}}$ are taken from measurements of $C(T, H)$ (triangles) and $\rho(T, H)$ (inverted triangles). The dashed line is an extrapolation of $T_{\mathrm{M}}$ to $0 \mathrm{~K}$. The color gradient represents $\partial \ln \left(\rho-\rho_{\mathrm{o}}\right) / \partial \ln T$, where $\rho_{\mathrm{o}}$ maximizes the $T$ range of the low $T$ power law behavior for each $\rho(T, H)$. The values of $\partial \ln \left(\rho-\rho_{\mathrm{o}}\right) / \partial \ln T$ above the low $T$ fit range do not represent the local exponent $n$ since they are based on $\rho_{\mathrm{o}}$ values from the low $T$ fits. Note that the low $T$ power law fit range appears to be reduced from what would be inferred from Fig. 4 (below). This is because the data are smoothed before taking $\partial \ln \left(\rho-\rho_{0}\right) / \partial \ln T$, resulting in a reduction of the apparent fit range because adjacent averaging samples the lower slope data above the upper $T$ limit of the fit range. After Ref. [21].

The classical QCP scenario is illustrated in Fig. 2 from Ref. [2] in which various quantum critical scenarios are depicted schematically: 1(a) - classical QCP scenario; 1(b) - unconventional QCP scenario where the NFL behavior extends far away from the QCP (or is not associated with a readily identifiable QCP); 1(c) and 1(d) - QCP enveloped by a superconducting "dome" for cases 1(a) and 1(b); 1(d) and 1(f) - superconducting region entirely within or outside of the ordered phase, respectively. All of these situations have been observed 
experimentally and are extensively discussed in Ref. [2]. In contrast to these phase diagrams, the NFL behavior for $\mathrm{Yb}_{2} \mathrm{Fe}_{12} \mathrm{P}_{7}$ extends far beyond the only possible QCP as $H$ is increased. In particular, we find: (1) a magnetically ordered ground state $\left(T_{\mathrm{M}} \sim 0.9 \mathrm{~K}\right)$ for which the signatures in electrical transport and thermal measurements are suppressed to lower $T$ with $H$; (2) a NFL ground state for $T<T_{\mathrm{M}}$ with a large $H=0 \mathrm{~T}$ electronic specific heat coefficient $\gamma$ that is suppressed with increasing $H$, and (3) a rapid crossover from the $H=$ $0 \mathrm{~T}$ NFL state to another extended NFL region which is increasingly stabilized for large values of $H$.
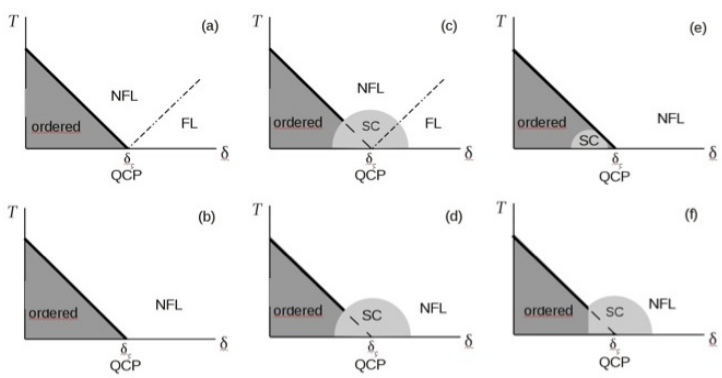

Fig. 2. Schematic temperature $T$ vs control parameter $\delta$ phase diagram for different NFL scenarios, where $\delta$ is usually chemical composition $x$, pressure $P$, or magnetic field $H$. The ordered state exhibiting FL behavior is suppressed when the control parameter $\delta$ is increased. At the critical value $\delta_{\mathrm{cr}}$, the order parameter vanishes at a quantum critical point (QCP). The phase diagrams illustrate the salient features of some of the many possible scenarios. Not every phase diagram has been observed in this exact form in real systems. Adapted from Ref. $[2]$.

Specific heat divided by temperature $C(T, H=0) / T$ data are shown in Fig. 3(d). $C(T, H=0) / T$ exhibits a broad peak near $120 \mathrm{~K}$ associated with the phonon contribution, below which $C(T, H=0) / T$ decreases down to $T=10 \mathrm{~K}$, passes through a minimum, and then increases rapidly, suggesting the formation of a strongly correlated electron ground state. A sharp peak in $C(T, H=0) / T$ is evident at $0.9 \mathrm{~K}$ [Fig. 3(b)], which indicates that $\mathrm{Yb}_{2} \mathrm{Fe}_{12} \mathrm{P}_{7}$ undergoes a phase transition near $T_{\mathrm{M}} \sim 0.9 \mathrm{~K}$. With an applied magnetic field $(H \perp \mathrm{c})$, this feature is completely suppressed by $H=1 \mathrm{~T}$, although the large background upon which it is superimposed is hardly affected. The suppression of $T_{\mathrm{M}}$ with $H$ suggests that the magnetic phase has an antiferromagnetic component. Below $T_{\mathrm{M}}$, $C(T, H=0) / T$ passes through a broad maximum reaching $\sim 3.4 \mathrm{~J} / \mathrm{mol} \mathrm{Yb} \mathrm{K}^{2}$ at $T \sim 0.27 \mathrm{~K}$, followed by a shallow minimum near $0.15 \mathrm{~K}$, after which, $C(T, H=0) / T$ increases again for $T<0.15 \mathrm{~K}$. For $H>1 \mathrm{~T}, C(T, H) / T$ is suppressed and the location of the broad maximum shifts towards higher $T$, consistent with the crossover to a FL state. The upturn in $C / T$ at the lowest temperatures is consistent with a nuclear Schottky anomaly as discussed in Ref. [21].

Electrical resistivity $\rho(T, H)$ data for $H \perp \mathrm{c}$ for $T=50 \mathrm{mK}$ $-300 \mathrm{~K}$ are shown in Fig. 4. The electrical resistivity $\rho(T, H=0)$ decreases from room temperature, displays a broad shoulder below $T \sim 50 \mathrm{~K}$, and then decreases rapidly below $\sim 2 \mathrm{~K}$ with a break in slope at $T_{\mathrm{M}} \sim 0.9 \mathrm{~K}$. It is notable that $\rho(T, H=0)$ is nearly linear for more than a decade in $T$ below $T_{\mathrm{M}}$ [Fig. 4(a)]. By applying $H \perp \mathrm{c}$, the maximum in $\partial \rho(T, H) / \partial T$ is suppressed and is no longer observable by $H \sim 0.7 \mathrm{~T}$. As expected for electron transport phenomena near a critical point where magnetic fluctuations are dominant, the shapes of $\partial \rho(T, H) / \partial T$ and $C(T) / T$ are similar and the resulting estimates for $T_{\mathrm{M}}$ from these two measurements are in good agreement. To characterize $\rho(T, H)$ at low $T$, the data were fitted using the expression $\rho(T)=\rho_{0}+A T^{n}$. The best fit was determined from a plot of $\ln \left(\rho-\rho_{\mathrm{o}}\right)$ vs $\ln (T)$ in which the value of $\rho_{\mathrm{o}}$ was selected to maximize the linear region of the fit extending from low $T$. Examples of these fits are shown in Fig. 4(b). The quantities $\rho_{0}, A$, and $n$ are plotted in Figs. 1(a)-(c).

The $C(T, H)$ and $\rho(T, H)$ measurements described here were used to construct the $T-H$ phase diagram shown in Fig. 1(d). The magnetic ordering temperature $T_{\mathrm{M}}$ decreases with $H$ to $\sim 0.7 \mathrm{~T}$, above which it can no longer be tracked. Thus, it is unclear whether there is a first order transition near $0.7 \mathrm{~T}$ or a second order transition in the vicinity of $\sim 1.5 \mathrm{~T}$, where we have extrapolated $T_{\mathrm{M}}=0$ $\mathrm{K}$ [dashed line in Fig. 1(d)]. The power law fits described above reveal that $n \sim 1.1$ for $H=0 \mathrm{~T}$. As $H$ is increased, $n$ rapidly crosses over to a value $\sim 1.5$ near $H=0.5 \mathrm{~T}$, close to the value of $H$ where $T_{\mathrm{M}}$ is no longer observable. Starting near $H \sim 2.5 \mathrm{~T}$, the $n \sim 1.5$ dependence extends over an increasingly broad $T$ region. The residual resistivity $\rho_{\mathrm{o}}$ increases and the prefactor $A$ decreases with increasing $H$.

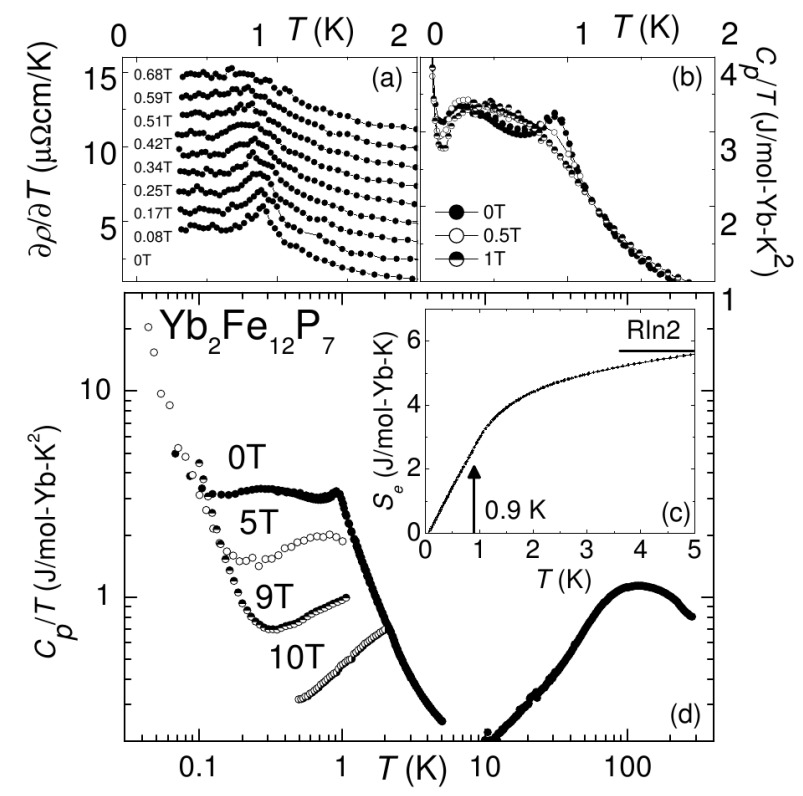

Fig. 3. (a) Derivative of the electrical resistivity with respect to temperature, $\partial \rho(T, H) / \partial T$. (b) Expanded view of $C(T, H) / T$ for $T<2 \mathrm{~K}$ and $H<1 \mathrm{~T}$. (c) Electronic portion of the entropy $S_{\mathrm{e}}(T, H=0)$ for $T<5 \mathrm{~K}$. (d) $C / T$ vs $T$ for several values of $H$. After Ref. [21]. 


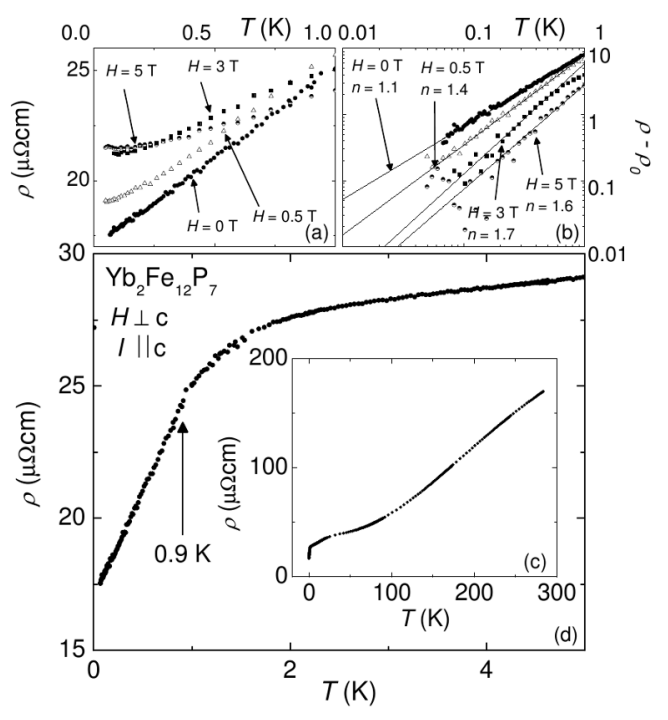

Fig. 4. (a) Electrical resistivity $\rho$ vs temperature $T$ for $0.05-$ $1 \mathrm{~K}$ and magnetic fields $H \perp \mathrm{c}$ axis. (b) $\rho-\rho_{\mathrm{o}}$ vs $T$ on a $\log -\log$ plot, fitted by straight lines as described in the text. (c) $\rho$ vs $T$ for $T=0.05-290 \mathrm{~K}$ and $H=0 \mathrm{~T}$. (d) $\rho$ vs $T$ for $T=0.05-5 \mathrm{~K}$ and $H=0$ T. After Ref. [21].

\subsection{Heavy fermion behavior and itinerant ferromagnetism in $\mathrm{Sm}_{2} \mathrm{Fe}_{12} \mathrm{P}_{7}$}

Measurements of $\rho, C$ and $M$ as a function of temperature and magnetic field on single crystals of $\mathrm{Sm}_{2} \mathrm{Fe}_{12} \mathrm{P}_{7}$ yielded evidence for heavy fermion behavior and itinerant ferromagnetism [22]. The $M(H, T)$ measurements demonstrate that $\mathrm{Sm}_{2} \mathrm{Fe}_{12} \mathrm{P}_{7}$ exhibits ferromagnetic order below $T_{\mathrm{M}, 1}=6.3 \mathrm{~K}$, as shown in Fig. 5 . The ratio of the effective magnetic moment in the paramagnetic state, obtained from a Curie-Weiss fit to the magnetic susceptibility, to the saturation magnetic moment in the ferromagnetically ordered state indicates that the ferromagnetic order involves itinerant electrons. The specific heat measurements reveal an enhanced value of the electronic specific heat coefficient $\gamma \sim 450 \mathrm{~mJ} / \mathrm{mol}-$ $\mathrm{K}^{2}$, as shown in Fig. 6, that is accompanied by a large coefficient $A$ of the $T^{2}$ term in the electrical resistivity at low temperatures, suggesting a heavy fermion ground state. Several consecutive magnetic phase transitions, indicative of competing magnetic energy scales and the observation of a metamagnetic transition in the magnetization data additionally suggest potential proximity to a quantum critical point.

\subsection{Actinide based compounds $\mathrm{U}_{2} \mathrm{Fe}_{12} \mathrm{P}_{7}$ and $\mathrm{Th}_{2} \mathrm{Fe}_{12} \mathrm{P}_{7}$}

Magnetization, specific heat, and electrical resistivity measurements [23] on single crystals of the compounds $\mathrm{U}_{2} \mathrm{Fe}_{12} \mathrm{P}_{7}$ and $\mathrm{Th}_{2} \mathrm{Fe}_{12} \mathrm{P}_{7}$ reveal that $\mathrm{U}_{2} \mathrm{Fe}_{12} \mathrm{P}_{7}$ displays antiferromagnetic order at a Néel temperature $T_{\mathrm{N}}=14 \mathrm{~K}$, while $\mathrm{Th}_{2} \mathrm{Fe}_{12} \mathrm{P}_{7}$ is a metal which exhibits Pauli paramagnetism with no evidence for superconductivity for $T \geq 1.1 \mathrm{~K}$. Magnetization measurements on $\mathrm{U}_{2} \mathrm{Fe}_{12} \mathrm{P}_{7}$

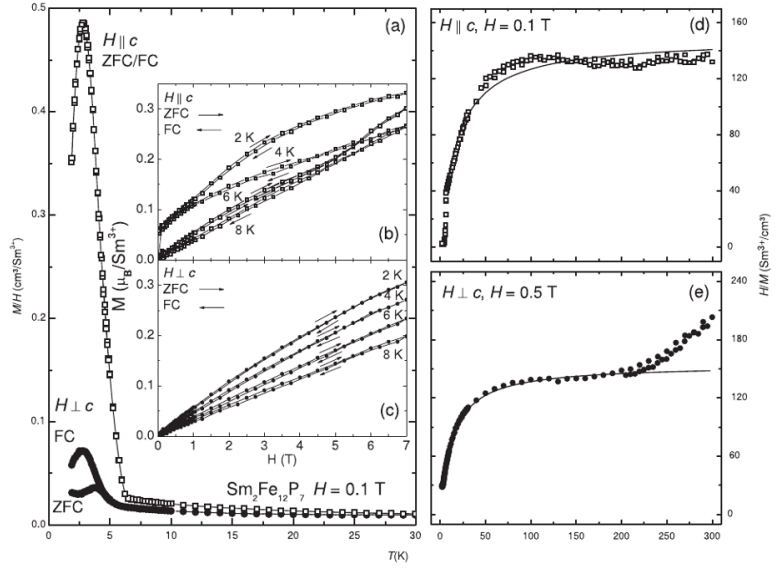

Fig. 5. Magnetization and magnetic susceptibility data for $\mathrm{Sm}_{2} \mathrm{Fe}_{12} \mathrm{P}_{7}$ single crystals. (a) Magnetic susceptibility $\chi=M / H$ vs temperature $T$ for magnetic field $H$ applied parallel $(\|)$ and perpendicular $(\perp)$ to the $\mathrm{c}$-axis. ZFC and FC indicate data taken after zero-field cooling and field cooling, respectively. (b) $M$ vs $H$ for $H \|$ c. (c) $M$ vs $H$ for $H \perp$ c. (d) and (e) Inverse magnetic susceptibility $\chi^{-1}=H / M$ vs temperature $T$ for $H \| \mathrm{c}$ and $H \perp \mathrm{c}$, respectively. Note that for $H \perp \mathrm{c}$, the measurement was carried out in $H=0.5 \mathrm{~T}$ instead of $0.1 \mathrm{~T}$ due to the weak response in this direction. The solid lines represent modified Curie-Weiss fits to the data as described in Ref. [11]. After Ref. [22].

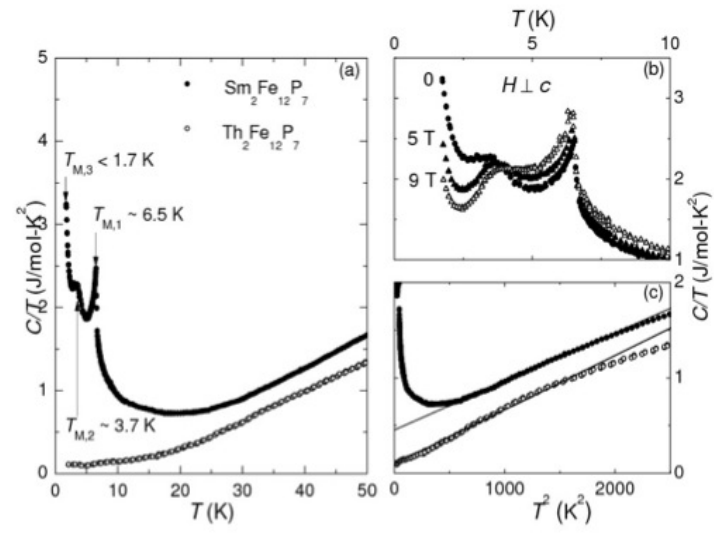

Fig. 6. (a) Specific heat $C$ divided by temperature $T$ for $\mathrm{Th}_{2} \mathrm{Fe}_{12} \mathrm{P}_{7}$ and $\mathrm{Sm}_{2} \mathrm{Fe}_{12} \mathrm{P}_{7}$ in applied magnetic field $H=0$. (b) $C / T$ for $\mathrm{Sm}_{2} \mathrm{Fe}_{12} \mathrm{P}_{7}$ for several values of $H$ applied perpendicular to the c-axis. The arrows and their corresponding labels $T_{\mathrm{M}, \mathrm{i}}$ refer to magnetic phase transitions. (c) $C / T$ vs $T^{2}$ for $\mathrm{Th}_{2} \mathrm{Fe}_{12} \mathrm{P}_{7}$ and $\mathrm{Sm}_{2} \mathrm{Fe}_{12} \mathrm{P}_{7}$. After Ref. [22].

show complicated magnetic behavior involving the $\mathrm{U}$ and, possibly, Fe ions, as well; e.g., hysteretic temperature and field dependences and metamagnetism. Electrical resistivity measurements on $\mathrm{U}_{2} \mathrm{Fe}_{12} \mathrm{P}_{7}$ also indicate large spin disorder scattering of conduction electrons for $T>T_{\mathrm{N}}$.

\subsection{Ferromagnetic ordering of $\mathrm{Co}$ in $\mathrm{Yb}_{2} \mathrm{Co}_{12} \mathrm{P}_{7}$}

One of the notable characteristics of $\mathrm{M}_{2} T_{12} \mathrm{Pn}_{7}$ compounds is that the transition metal sublattice can be tuned from non-magnetic ( $T=\mathrm{Fe}$ or Ni) to magnetic ( $T=$ 
Co) [17, 24-28]. We recently performed measurements of $M(T, H)$ and $C(T)$ to $2 \mathrm{~K}$ and $\rho(T)$ to millikelvin temperatures on polycrystalline samples of $\mathrm{Yb}_{2} \mathrm{Co}_{12} \mathrm{P}_{7}$. In addition to the previously reported ferromagnetic ordering of the cobalt sublattice at $T_{\mathrm{C}}=136 \mathrm{~K}$, we found an additional magnetic transition below $T_{\mathrm{M}}=5 \mathrm{~K}$ that is likely associated with ordering of the $\mathrm{Yb}$ ions. The broad nature of the specific heat anomaly suggests disordered magnetism and possible short-range correlations well above $T_{\mathrm{M}}$.

\section{Emergence of superconductivity upon suppression of charge density wave ordering in the rare earth tritellurides $\mathrm{RTe}_{3}$}

The quasi-2D rare-earth tritelluride compounds $R \mathrm{Te}_{3}(R=$ La-Nd, Sm, Gd-Tm) have received appreciable attention since they constitute the first system in which nominal square-planar symmetry is broken by the formation of a unidirectional charge density wave (CDW) [29]. Furthermore, the CDW transition temperatures of these compounds display striking systematic behavior within the rare-earth series [30-33]. The rare-earth tritellurides crystallize in the $\mathrm{NdTe}_{3}$ structure that belongs to the space group Cmcm (No. 63); the structure consists of alternating double layers of nearly square-planar $\mathrm{Te}$ sheets and corrugated double $R$ Te layers and forms a weakly orthorhombic lattice above the CDW transition temperature [34]. In this standard space group denomination, the b-axis is oriented perpendicular to the Te layers, and the average lattice parameters for all the lanthanide series are $(a, b, c)=(4,26,4) \AA$. Because large areas of Fermi surface are parallel and may be connected by a single nesting vector, these compounds are unstable to the formation of an incommensurate CDW within the ac-planes. The modulation of the lattice is characterized by a single in-plane wave vector $q_{1}$, which has approximately the same value for all the rare earths $\left(q_{1} \approx 2 \mathrm{c}^{*} / 7\right.$, with $\left.\mathrm{c}^{*}=2 \pi / \mathrm{c}\right)$ [30]. For tritellurides containing the heavier rare earths ranging from Dy to Tm, a second $\mathrm{CDW}$, characterized by a wave vector $q_{2}$ transverse to the first one, and of larger value $\left(q_{2} \approx \mathrm{a}^{*} / 3\right)$, forms at lower temperatures [32]. These compounds also display magnetic order at or below $\sim 10 \mathrm{~K}[33,35]$. Electronic structure calculations and ARPES measurements indicate that the $R T$ e layers play little or no role in the Fermi surface which is determined instead by the Te sheets [36-38]. Therefore, rare earth substitution may be viewed as applying "chemical pressure" in that it primarily alters the lattice parameters without affecting the band filling or structure type [30]. The CDW transition temperatures of the $R \mathrm{Te}_{3}$ compounds have been found to correlate well with the in-plane lattice parameters.

Motivated by the correspondence between the CDW transition temperatures and the lattice parameters ("chemical pressure") of the $R \mathrm{Te}_{3}$ compounds, we embarked on a study of these compounds under pressure to determine the pressure dependence of the CDW and $R$ ion magnetic ordering temperatures and search for the possible emergence of superconductivity, in the event that any of these ordering temperatures could be driven towards $0 \mathrm{~K}$ under pressure. The compound $\mathrm{TbTe}_{3}$ was selected for the first experiments since it appeared that a small reduction in lattice parameter could induce a second CDW which might lead to unusual phases if the CDW and long-range $R$ ion magnetic ordering temperatures could be brought into coincidence. This was followed by investigations of the compounds $\mathrm{CeTe}_{3}$, $\mathrm{GdTe}_{3}$ and $\mathrm{DyTe}_{3}$. The CDW, magnetic, and superconducting transitions were tracked as a function of pressure from electrical resistivity $\rho(\mathrm{T})$ measurements under pressure using piston-cylinder and Bridgman anvil clamped high pressure cells on single crystal specimens of all of the $R \mathrm{Te}_{3}$ samples investigated [39-41].

The initial high pressure experiments on $\mathrm{TbTe}_{3}$ revealed that the upper $\mathrm{CDW}$ transition temperature $T_{\mathrm{CDW} 1}$, inferred from a feature in $\rho(T)$, was suppressed with pressure. A second feature in $\rho(T)$ appeared above $1.2 \mathrm{GPa}$ and moved to higher temperatures with pressure, consistent with the appearance of a second, lower temperature, $\mathrm{CDW}$, with a transition temperature $T_{\mathrm{CDW} 2}$. The pressure dependence of $\rho(T)$ of $\mathrm{TbTe}_{3}$ measured in the piston-cylinder pressure cell to $\sim 2.3 \mathrm{GPa}$, is shown in Fig. 7. Above $2.3 \mathrm{GPa}$, we found that $\mathrm{TbTe}_{3}$, becomes superconducting with a $T_{\mathrm{c}}(50 \%$ drop of transition in electrical resistivity) reaching $\sim 3.5 \mathrm{~K}$ near $12 \mathrm{GPa}$. Interestingly, at $2.3 \mathrm{GPa}$, three types of order, CDW, $R$ ion antiferromagnetism (AFM), and superconductivity, all appear upon progressively cooling the sample.

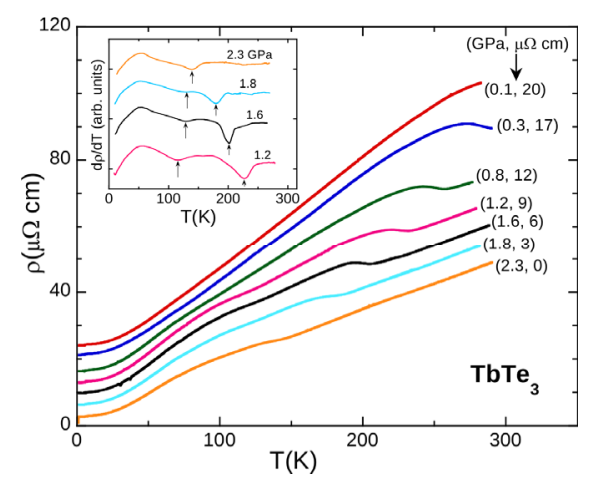

Fig. 7. Electrical resistivity measured in the ac-plane of $\mathrm{TbTe}_{3}$ under nearly hydrostatic pressures to $2.3 \mathrm{GPa}$. The curves have been offset vertically for clarity. The pressure and magnitude of the offset are indicated in parentheses at the right of the curves. The onset of the upper CDW is clearly seen to shift downward with pressure. At and above $1.2 \mathrm{GPa}$, a second feature in $\rho(T)$, apparently associated with the lower CDW, is clearly visible as a minimum in the slope $\mathrm{d} \rho / \mathrm{d} T$ (inset). At $2.3 \mathrm{GPa}$, the two transitions $T_{\mathrm{CDW} 1}$ and $T_{\mathrm{CDW} 2}$ appear to be nearly coincident. After Ref. [42].

Experiments on $\mathrm{GdTe}_{3}$ and $\mathrm{DyTe}_{3}$ yielded results similar to those found for $\mathrm{TbTe}_{3}$, in which the application of pressure was found to suppress $T_{\mathrm{CDW} 1}$ of the upper $\mathrm{CDW}$ and increase $T_{\mathrm{CDW} 2}$ of the lower $\mathrm{CDW}$, until it appeared to merge with $T_{\mathrm{CDW} 1}$, and induce 
superconductivity with a $T_{\mathrm{c}} \sim 1 \mathrm{~K}$ at $\sim 3.0 \mathrm{GPa}$. Measurements were also extended to lower temperatures $(\sim 40 \mathrm{mK})$ in the lower pressure region, which revealed that superconductivity occurs at pressures as low as $1.2 \mathrm{GPa}$ with $T_{\mathrm{c}}$ values of the order of $300 \mathrm{mK}$. The $T-$ $P$ phase diagrams derived from the $\rho(\mathrm{T})$ measurements on $\mathrm{GdTe}_{3}, \mathrm{TbTe}_{3}$, and $\mathrm{DyTb}_{3}$ under pressure, plotted on logarithmic temperature and linear pressure scales, are very similar, as shown in Fig. 8. The figure shows that the upper CDW transition temperatures are suppressed in a similar manner and apparently vanish in the neighborhood of $3-4 \mathrm{GPa}$. Actually, $T_{\mathrm{CDW} 1}$ decreases linearly with pressure for $\mathrm{GdTe}_{3}, \mathrm{TbTe}_{3}$, and $\mathrm{DyTe}_{3}$ at a rate $\mathrm{d} T_{\mathrm{CDW} 1} / \mathrm{d} P \approx-7 \mathrm{~K} / \mathrm{kbar}$ and extrapolates to $0 \mathrm{~K}$ at pressures of $\sim 4.7,4.3$, and $3.8 \mathrm{GPa}$, respectively. The $R$ ion magnetic ordering temperatures of the $\mathrm{TbTe}_{3}$ and $\mathrm{DyTe}_{3}$ compounds, which can be determined from small features in $\rho(\mathrm{T})$ under the nearly hydrostatic conditions employed in the piston-cylinder clamps, increase slightly with pressure and cannot be followed above $\sim 2.5 \mathrm{GPa}$ using the less hydrostatic conditions encountered in the Bridgman anvil high pressure clamps. The situation with the superconductivity measurements is more complicated in that elemental $\mathrm{Te}$ is known to exhibit superconductivity when it is metalized at high pressures above $4 \mathrm{GPa}$. The dependence of the $T_{\mathrm{c}}$ of elemental Te on pressure is indicated by the solid black line in Fig. 8, which resembles the variation of $T_{\mathrm{c}}$ with pressure determined from the measurements on the $\mathrm{RTe}_{3}$ compounds. Thus, one possibility is that the behavior of $T_{\mathrm{c}}$ above $4 \mathrm{GPa}$ inferred from the $\rho(T, P)$ measurements on the $R \mathrm{Te}_{3}$ compounds is extrinsic and due to inclusions of Te in the flux grown single crystals. However, various analytical studies have not revealed any evidence of $\mathrm{Te}$ inclusions in the $R \mathrm{Te}_{3}$ crystals and ac magnetic susceptibility measurements at low temperatures and pressures up to $2.5 \mathrm{GPa}$ indicate that the superconductivity in this region exhibits full shielding. Given the ac magnetic susceptibility results and the fact that elemental $\mathrm{Te}$ is insulating below $\sim 4 \mathrm{GPa}$, the superconductivity in the low-pressure/low-temperature region of the $T-P$ phase diagram appears to be intrinsic. Thus, there presently appear to be two scenarios, both of which involve a "dome" of superconductivity in the neighborhood of the critical pressure $P_{\text {cr }}$ where the CDW transitions are suppressed which overlaps $P_{\mathrm{cr}}$, resulting in a region were the CDW order, $R$ ion AFM order, and superconductivity all coexist. In the first scenario, the superconducting dome is wide (blue and orange colored regions labelled $\mathrm{SC} 1$ and $\mathrm{SC} 2$ in the figure), whereas in the second scenario, the "dome" is narrow (blue region labelled SC1 in the figure), with the SC2 phase arising due to inclusions of Te. Further experiments are underway to distinguish between these possibilities. It is interesting that a similar $T-P$ phase diagram, in which a dome of superconductivity overlaps the pressure at which the CDW is suppressed, has been reported for the compound $1 T$-TiSe2 [43].

Finally, experiments on the $\mathrm{CeTe}_{3}$ system were undertaken, motivated, in part, by the fact that ceriumbased compounds frequently display an enhancement of

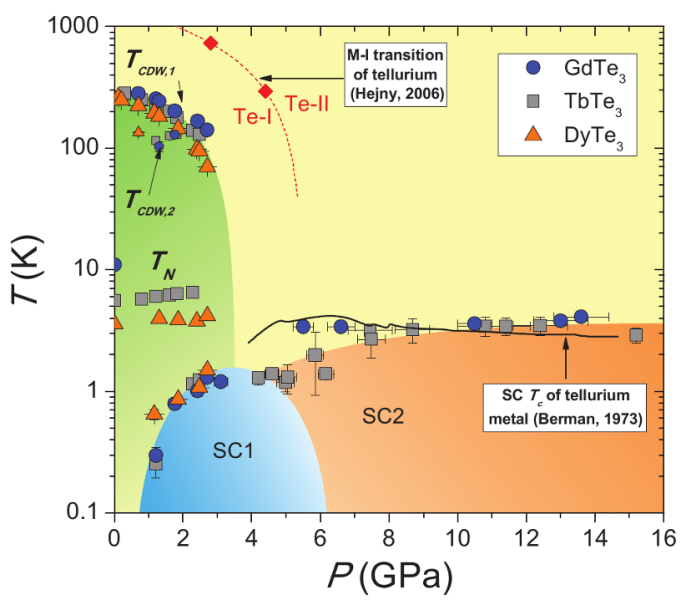

Fig. 8. Temperature - pressure $(T-P)$ phase diagram for superconducting $R \mathrm{Te}_{3}$ compounds. The charge density wave transition temperature $T_{\mathrm{CDW} 1}$ is suppressed with pressure, and its corresponding feature can no longer be distinguished from our measurements above $2.7 \mathrm{GPa}$. At intermediate temperatures, ordering of the rare-earth ions occurs at $T_{\mathrm{N}}$. At lower temperatures, a superconducting phase appears at $1.2 \mathrm{GPa}$ and $T_{\mathrm{c}} \sim 0.3 \mathrm{~K}$, where $T_{\mathrm{c}}$ reaches a maximum value of $3.5 \mathrm{~K}$ at 12 $\mathrm{GPa}$. The possible origins of the superconducting phases are discussed in the text. The black line corresponds to $T_{\mathrm{c}}(P)$ data for elemental Te taken from Ref. [44]; the red diamonds represent metal-insulator transitions and are from Ref. [45].

their electronic effective mass at low temperatures caused by the strong hybridization of the localized $4 f$ and conduction electron states and exhibit a variety of correlated electron ground states, such as localized moment magnetic order and superconductivity with many of these phases induced at high pressures. The competing interaction of the CDW with some of these strongly correlated electron states, "tuned" by varying chemical composition, pressure, or magnetic field, is of particular interest in these types of materials. We performed high pressure electrical transport measurements on $\mathrm{CeTe}_{3}$, along with a subkelvin specific heat experiment at ambient pressure and high magnetic fields [39]. We found that two magnetic phases occur below $20 \mathrm{~K}$, with nonparallel magnetic easy axes, inferred from additional transport measurements made in fields for different angles.

\section{Concluding remarks}

In this paper, we have briefly reviewed experiments performed in our laboratory that were designed to explore the types of phenomena that are found when spin or charge ordered phases are suppressed toward $0 \mathrm{~K}$ through the variation of an external control parameter such as chemical composition, pressure or magnetic field. We focused on examples within two systems of materials that we have been investigating during the past several years, the noncentrosymmetric $M_{2} T_{12} \mathrm{P}_{7} \quad(M=$ rare earth, actinide; $T=\mathrm{Co}, \mathrm{Fe})$ compounds and the $R \mathrm{Te}_{3}(R=\mathrm{Ce}$, $\mathrm{Gd}, \mathrm{Tb}, \mathrm{Dy})$ compounds, with emphasis on single crystal specimens. Experiments on the $M_{2} T_{12} \mathrm{P}_{7}$ compounds revealed heavy fermion behavior and itinerant 
ferromagnetism for $\mathrm{Sm}_{2} \mathrm{Fe}_{12} \mathrm{P}_{7}$, antiferromagnetic order and unconventional quantum critical behavior in a magnetic field for $\mathrm{Yb}_{2} \mathrm{Fe}_{12} \mathrm{P}_{7}$, Fermi liquid behavior for $\mathrm{Th}_{2} \mathrm{Fe}_{12} \mathrm{P}_{7}$, and complex antiferromagnetic order for $\mathrm{U}_{2} \mathrm{Fe}_{12} \mathrm{P}_{7}$. In contrast, $\mathrm{Yb}_{2} \mathrm{Co}_{12} \mathrm{P}_{7}$ displays ferromagnetic ordering of the cobalt sublattice at $T_{\mathrm{C}}=136 \mathrm{~K}$ and a transition below $T_{\mathrm{M}}=5 \mathrm{~K}$ that is likely due to magnetic ordering of the $\mathrm{Yb}$ ions. Investigations of the $R \mathrm{Te}_{3}$ compounds under pressure uncovered the emergence of superconductivity in a "dome" shaped region near the critical pressure $P_{\text {cr }} \approx 3-4 \mathrm{GPa}$ at which the CDW is suppressed, with coexistence of superconducting and $\mathrm{CDW}$ order for pressures below $P_{\mathrm{cr}}$, for $R=\mathrm{Gd}, \mathrm{Tb}$, and $\mathrm{Dy}$, and the interplay of CDW and Ce antiferromagnetic order for $R=$ Ce.

\section{Acknowledgements}

The authors gratefully acknowledge the support of the US Department of Energy under Grant No. DE-FG02-04ER46105 (funding materials synthesis and characterization) and Grant No. DE-FG52-09NA29459 (funding high pressure measurements), US National Science Foundation under Grant No. DMR-0802478 (funding low temperature measurements), and the US Air Force Office of Scientific Research - Multidisciplinary University Research Initiative under Grant No. FA955009-1-0603 (funding superconductivity searches). M. J. gratefully acknowledges financial support from the Alexander von Humboldt foundation.

\section{References}

1. M. B. Maple, R. E. Baumbach, J. J. Hamlin, D. A. Zocco, B. J. Taylor, N. P. Butch, J. R. Jeffries, S. T. Weir, B. C. Sales, D. Mandrus, M. A. McGuire, A. S. Sefat, R. Jin, Y. K. Vohra, J.-H. Chu, and I. R. Fisher, Physica B 404, 2924 (2009)

2. M. B. Maple, R. E. Baumbach, N. P. Butch, J. J. Hamlin, and M. Janoschek, J. Low Temp. Phys. 161, 4 (2010)

3. C. L. Seaman, M. B. Maple, B. W. Lee, S. Ghamaty, M. S. Torikachvili, J.-S. Kang, L. Z. Liu, J. W. Allen, and D. L. Cox, Phys. Rev. Lett. 67, 2882 (1991)

4. M. B. Maple, C. L. Seaman, D. A. Gajewski, Y. Dalichaouch, V. B. Barbetta, M. C. de Andrade, H. A. Mook, H. G. Lukefahr, O. O. Bernal, and D. E. MacLaughlin, J. Low Temp. Phys. 95, 225 (1994)

5. M. B. Maple, M. C. de Andrade, J. Herrmann, Y. Dalichaouch, D. A. Gajewski, C. L. Seaman, R. Chau, R. Movshovich, M. C. Aronson, and R. Osborn, J. Low Temp. Phys. 99, 223 (1995)

6. P. Coleman, M. B. Maple, and A. J. Millis (eds.), J. Phys.: Cond. Matt. 8 (See various articles in Proceedings of the Institute for Theoretical Physics Conference on Non-Fermi Liquid Behavior in Metals, Santa Barbara) (1996)

7. G. R. Stewart, Rev. Mod. Phys. 78, 743 (2006)

8. H. v. Löhneysen, A. Rosch, M. Vojta, and P. Wölfle, Rev. Mod. Phys. 79, 1015 (2007)
9. Q. Si and F. Steglich, Science 329, 1161 (2010)

10. H. Q. Yuan, D. F. Agterberg, N. Hayashi, P. Badica, D. Vandervelde, K. Togano, M. Sigrist, and M. B. Salamon, Phys. Rev. Lett. 97, 017006 (2006)

11. S. Mühlbauer, B. Binz, F. Jonietz, C. Pfleiderer, A. Rosch, A. Neubauer, R. Georgii, and P. Böni, Science 323, 915 (2009)

12. I. E. Dzyaloshinskii, J. Phys. Chem Solids 4, 241 (1958)

13. T. Moriya, Phys. Rev. 120, 91 (1960)

14. C. Pfleiderer, A. Neubauer, S. Mühlbauer, F. Jonietz, M. Janoschek, S. Legl, R. Ritz, W. Münzer, C. Franz, P. G. Niklowitz, T. Keller, R. Georgii, P. Böni, B. Binz, A. Rosch, U. K. Rößler, and A. N. Bogdanov, J. Phys.: Cond. Matt. 21, 164215 (2009)

15. J. J. Hamlin, R. E. Baumbach, D. A. Zocco, T. A. Sayles, and M. B. Maple, J. Phys.: Cond. Matt. 20, 365220 (2008)

16. E. Ganglberger, Monatsh. Chem. 99, 557 (1968)

17. W. Jeitschko, D.J. Braun, R.H. Ashcraft and R. Marchand, J. Solid State Chem. 25, 309 (1978)

18. W. Jeitschko, P. Pollmeier and U. Meisen, J. Alloys Compds. 196, 105 (1993)

19. A. Hellmann and A. Mewis, Z. Anorg. Allg. Chem. 627, 1357 (2001).

20. Y. M. Prots and W. Jeitschko, Inorg. Chem. 37, 5431 (1998).

21. R. E. Baumbach, J. J. Hamlin, L. Shu, D. A. Zocco, J. R. O'Brien, P.-C. Ho, and M. B. Maple, Phys. Rev. Lett. 105, 106403 (2010)

22. M. Janoschek, R. E. Baumbach, J. J. Hamlin, I. K. Lum, and M. B. Maple, J. Phys.: Cond. Matt. 23, 094221 (2011)

23. R. E. Baumbach, J. J. Hamlin, M. Janoschek, I. K. Lum, and M. B. Maple, J. Phys.: Cond. Matt. 23, 094222 (2011)

24. M. Reehuis and W. Jeitschko, J. Phys. Chem. Solids 50, 563 (1989)

25. E. H. El Ghadraoui, J. Y. Pivan, O. Pena, R. Guerin, and P. Bonville, Physica B 163, 185 (1990)

26. H. Raffius, E. Mörsen, B. D. Mosel, W. MüllerWarmuth, T. Hilbich, M. Reehuis, T. Vomhof, and W. Jeitschko, J. Phys. Chem. Solids 52, 787 (1991)

27. K. Zeppenfeld and W. Jeitschko, J. Phys. Chem. Solids 54, 1527 (1993)

28. M. Reehuis, N. Stüßer, A. Nientiedt, T. Ebel, W. Jeitschko, and B. Ouladdiaf, J. Magn. Magn. Mater. 177, 805 (1998)

29. A. Fang, N. Ru, I. R. Fisher, and A. Kapitulnik, Phys. Rev. Lett. 99, 046401 (2007)

30. E. DiMasi, M. C. Aronson, J. F. Mansfield, B. Foran, and S. Lee, Phys. Rev. B 52, 14516 (1995)

31. A. Sacchetti, L. Degiorgi, T. Giamarchi, N. Ru, and I. R. Fisher, Phys. Rev. B 74, 125115 (2006)

32. N. Ru, C. L. Condron, G. Y. Margulis, K. Y. Shin, J. Laverock, S. B. Dugdale, M. F. Toney, and I. R. Fisher, Phys. Rev. B 77, 035114 (2008)

33. N. Ru, J. H. Chu, and I. R. Fisher, Phys. Rev. B 78, 012410 (2008)

34. B. K. Norling and H. Steinfink, Inorg. Chem. 5, 1488 (1966) 
35. Y. Iyeiri, T. Okumura, C. Michioka, and K. Suzuki, Phys. Rev. B 67, 144417 (2003)

36. H. Komoda, T. Sato, S. Souma, T. Takahashi, Y. Ito, and K. Suzuki, Phys. Rev. B 70, 195101 (2004)

37. J. Laverock, S. B. Dugdale, Z. Major, M. A. Alam, N. Ru, I. R. Fisher, G. Santi and E. Bruno, Phys. Rev. B 71, 085114 (2005)

38. V. Brouet, W. L. Yang, X. J. Zhou, Z. Hussain, R. G. Moore, R. He, D. H. Lu, Z. X. Shen, J. Laverock, S. B. Dugdale, N. Ru, and I. R. Fisher, Phys. Rev. B 77, 235104 (2008)

39. D. A. Zocco, J. J. Hamlin, T. A. Sayles, M. B. Maple, J.-H. Chu and I. R. Fisher, Phys. Rev. B 79, 134428 (2009)

40. D. A. Zocco, Ph.D. Thesis, University of California, San Diego (2011)

41. D. A. Zocco, J. J. Hamlin, M. B. Maple, J.-H. Chu, and I. R. Fisher, to be published

42. J. J. Hamlin, D. A. Zocco, T. A. Sayles, M. B. Maple, J.-H. Chu, and I. R. Fisher, Phys. Rev. Lett. 102, 177002 (2009)

43. A. F. Kusmartseva, B. Sipos, H. Berger, L. Forro, and E. Tutis, Phys. Rev. Lett. 103, 236401 (2009)

44. I. V. Berman, Zh. I. Binzarov, and P. Kurkin, Fiz. Tverd. Tela. 14, 2527 (1972); Sov. Solid State 14, 2192 (1973)

45. C. Hejny, S. Falconi, L. F. Lundegaard, and M. I. McMahon, Phys. Rev. B 74, 174119 (2006) 